Research Article

\title{
Synthesis and Characterization of CeGdZn-Ferrite Nanoparticles as Magnetic Hyperthermia Application Agents
}

\author{
Saleh S. Hayek (iD \\ Mechanical Engineering Department, Faculty of Engineering, University of Tabuk, P.O. Box 741, Tabuk-71491, Saudi Arabia \\ Correspondence should be addressed to Saleh S. Hayek; sshayek@ut.edu.sa
}

Received 7 October 2019; Accepted 13 November 2019; Published 27 November 2019

Academic Editor: Luigi Nicolais

Copyright (c) 2019 Saleh S. Hayek. This is an open access article distributed under the Creative Commons Attribution License, which permits unrestricted use, distribution, and reproduction in any medium, provided the original work is properly cited.

\begin{abstract}
This study focuses on the synthesis and characterization of a magnetic nanomaterial used in magnetic hyperthermia. Cerium gadolinium zinc-iron (CeGdZnFe) magnetic nanoparticles were synthesized by a coprecipitation method for application as magnetic hyperthermia agents. Determination of phase purity and their identification was achieved by X-ray diffraction studies using a Phillips powder diffractometer with $\mathrm{Cu}$ Ka radiation. Typical TEM micrographs of the dispersion of CeGdZn-ferrite nanoparticles and CeGdZn-ferrite PEG-encapsulated nanoparticles in ethanol deposited over a Cu grid were taken as part of the characterization techniques to be used for newly developed materials. It was then fitted by a Gaussian distribution with mean diameter $\mathrm{dm} \pm 1.0 \mathrm{~nm}$. The investigation of magnetic properties showed that adjusting, $\mathrm{Gd}$ and $\mathrm{Zn}$ contributes to the nanoparticles added to the adjustment of all magnetic properties of CeGdZnFe.
\end{abstract}

\section{Introduction}

Various studies have highlighted the colloidal nanoparticle synthesis advancement, which has enabled the implementation of various high-quality nanoparticles for treatment of various patients [1]. Primarily, the application of magnetic nanoparticles is observed as a promising tool for magnetic hyperthermia. Hyperthermia refers to a cancer therapy which uses heat treatment approach due to the vulnerability of the tumor cells to high temperatures [2]. The reduction in cancerous cell viability occurs along with an increase in their sensitivity to the chemotherapy as well as radiation, where the targeted tissue temperature rises to $42-46^{\circ} \mathrm{C}$ [3]. Most studies confirm the potential of cancer cells to be destroyed at $43^{\circ} \mathrm{C}$, while the survival tendency of normal cells ranges up to $46^{\circ} \mathrm{C}$. The methods applied for hyperthermia constitute capacitive heating, hot water, and inductive heating of malignant cells $[2,3]$. The possibility that artificially induced hyperthermia can facilitate the tumor treatment has increased the initiatives induced for developing different devices $[4,5]$. Despite the established efficacy of hyperthermia devices, its therapeutic efficiency has only been demonstrated for animals [6,7], with limited research on its application on individuals [4].

Initially, the magnetic particle hyperthermia heating treatment using magnetic nanoparticles was brought forward by Gilchrist et al. in 1957, which continued to be an active area of cancer research [8]. Bessalova et al. [9] have shown its diverse application for nano- and microsized object applications. Magnetic hyperthermia is based on the intratumoral deposition of biocompatible magnetic nanoparticles followed by exposure to a high frequency electromagnetic field [10]. The dissipation of energy resulted in local heating of the active particles that eventually leads to the death of cancer cells. The viability of cancer cells reduces and their sensitivity to chemotherapy and radiation also reduces, when malignant cells are heated to temperatures between 41 and $46^{\circ} \mathrm{C}$ [11-14]. However, it is found technologically challenging to control the on-site temperature increase for application of magnetic hyperthermia therapies.

Magnetic hyperthermia supplies heat at the tumor site by application of an external alternating magnetic field to the 
nanomagnetic particles. The particles then heat up and conduct that heat to the tumor cells. The materials' use with the Curie temperature ranging from 41 to $46^{\circ} \mathrm{C}$ protects against overheating of normal cells, due to the decrease of magnetic coupling in the paramagnetic regime [15]. It is because it uses the external magnetic field for binding of proteins, enzymes, and drugs as well as antibodies with tissues, tumor, or more [16]. Kmita et al. [17] have shown an increased interest for the ferrite nanoparticles using controllable morphology and crystal structure based on their escalated application in fundamental as well as industrial use. Different forms of nanoparticles such as nanorods, spherical, nanoflowers, and nanotubes along with their specific surface area facilitate alteration of their functional properties for applications [18, 19]. Various studies have shown ferrite nanoparticles' potential applications in biomedical applications $[20,21]$. It is because the fundamental electrical and magnetic properties of the ferrite can be changed for its desired application, where the use of zinc $(\mathrm{Zn})$ as a divalent cation is substantial [22-24].

Magnetic nanoparticle materials have high specific power loss, and suitable temperature dependence of power loss is allowed by an adjustment of the Curie temperature to $43^{\circ} \mathrm{C}$. One way to achieve this is through the use of magnetic nanoparticles with properties suitably modified by compositional variations. The most successful type which has been widely investigated consists of superparamagnetic iron oxide nanoparticles [25, 26]. Moreover, various iron oxidebased nanoparticles such as $\mathrm{XFe}_{2} \mathrm{O}_{4}(X=\mathrm{Co}, \mathrm{Ni}, \mathrm{Mn}, \mathrm{Zn}, \mathrm{Cu}$, $\mathrm{Mg}$, etc.) in [27] have also been developed and investigated for hyperthermia. Till now, magnetic iron oxide-based nanoparticles are predominantly used as a heating mediator in hyperthermia. Currently, magnetic nanoparticles are commercially supplied by Bayer Schering, Chemicell, and Micromod [28]. Generally, there are two steps to fabricate nanomagnetic particles for magnetic hyperthermia application, such as synthesis of inorganic nanomagnetic particles and encapsulation of nanomaterials by a polymer or with a suitable surfactant. In the first step, nanoparticles are formed by wet chemical methods such as chemical coprecipitation [29-31], borohydride reduction [32, 33], sonochemical method [34-36], vapor trapping [28], and molecular selfassembly. Dry methods may also be used for this purpose, such as laser ablation, microwave plasma vapor deposition [30], and carbon arc process [36].

Hoque et al. [24] and Guo et al. [25] have demonstrated significant attention to the zinc ferrite nanoparticles due to its reduced toxicity, particularly Gd-Zn [37]. Gd-Zn ferrite has recently gained much interest mainly because of the ability to change its properties by varying the constituent's proportions; however, the work on it remains limited. The study is derived as magnetic nanoparticles exposed to an alternating magnetic field are supposed to act as localized heat sources at certain target regions inside the human body. This allows dissipation of heat to the surrounding areas using a different magnetic field, allowing instigation of selfcontrolled magnetic hyperthermia therapy.

In recent years, many studies have been focused on optimizing the heating efficiency in terms of nanoparticle intrinsic properties such as particle size, anisotropy constant, saturation magnetization, easy axis orientations, extrinsic properties such as AC field frequency, and amplitude and the role of dipolar interactions. Gadolinium and cerium present several of those characteristics. They are easy to produce; they present very small hydrodynamic diameters, and they are biodegradable. Cerium oxide nanoparticles with integrated gadolinium exhibit combined therapeutic capabilities. This type of nanomaterial is highly promising for applications in the fields of biomedical magnetic hyperthermia. Therefore, the present study has synthesized CeGdZn-ferrite nanoparticles using the chemical coprecipitation method in which the proportions of zinc and cerium were varied, and the effects on the properties have been studied. The self-heating temperature-rising characteristics of CeGdZn-ferrite nanoparticles obtained by the coprecipitation process were analysed under different applied magnetic fields and frequencies to confirm their effectiveness as hyperthermia agents. The Curie temperatures of their particles were $\sim 315 \mathrm{~K}$.

The present study has reported on newly fabricated, assynthesized, self-heating magnetic nanoparticles, known as CeGdZnFe in magnetic hyperthermia. The CeGdZnFe nanoparticle system showed both superparamagnetic and ferromagnetic behaviors depending on their particle sizes. Materials having large heating power generation per particle unit mass are mostly applicable for hyperthermia. Therefore, the study has developed various types of magnetic nanoparticles that are used as magnetic mediators. In recent years, many studies have been focused on optimizing the heating efficiency in terms of nanoparticle intrinsic properties such as particle size, anisotropy constant, saturation magnetization, easy axis orientations, extrinsic properties such as AC field frequency, and amplitude and the role of dipolar interactions. Gadolinium and cerium present several of those characteristics. They are easy to produce; they present very small hydrodynamic diameters, and they are biodegradable.

\section{Materials and Methods}

Chemicals were used for encapsulated magnetic particles. Magnetic nanoparticles that possess high magnetic susceptibility and saturation magnetization were suitable candidates for magnetic hyperthermia medical application. The phases of CeGdZnFe nanoparticles purchased separately from Chemicell were synthesized by coupling of chemical coprecipitation and ultrasonication. The particles were further coated with a cationic surfactant. Many particles were very well dispersed and were in the size range of 6$10 \mathrm{~nm}$, as it is the case of as-synthesized CeGdZn-ferrites (Figure 1).

2.1. Synthesis of GdZnCe-Ferrite Magnetic Nanoparticles. In this study, several CeGdZn-ferrite particles with various constituent proportions were synthesized to check the possibility of decreasing the Curie temperature to $315 \mathrm{~K}$ by adding Ce. Several samples of CeGdZnFe were synthesized 


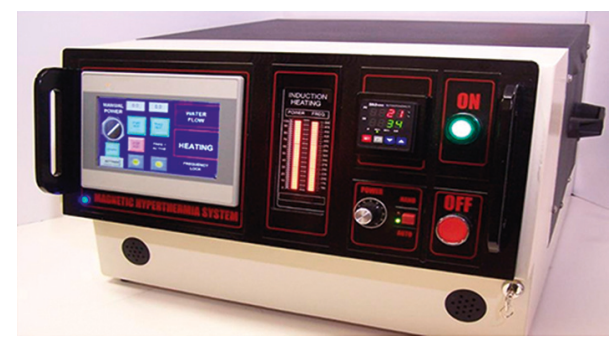

FIgURE 1: Magnetic hyperthermia apparatus [5].

using a chemical co-precipitation method. In this method, a $0.1 \mathrm{M}$ solution of the metal salts $\mathrm{GdC}_{12}, \mathrm{Fe}_{2} \mathrm{SO}_{4}, \mathrm{CeCl}_{3}$, and $\mathrm{ZnSO}_{4}$ was added to an $8 \mathrm{M}$ solution of $\mathrm{NaOH}$. The mixture was stirred vigorously at $90^{\circ} \mathrm{C}$ for 40 minutes. Thereafter, the synthesized magnetic nanoparticles were filtered and washed three times with distilled water and three times with acetone. The particles were then allowed to dry in air at room temperature.

\subsection{Ethyl Cellulose-Encapsulated CeGdZnFe using Polymer} Emulsion. The polymer emulsion method was used to encapsulate CeGdZn-ferrite nanoparticles inside ethyl cellulose (chemical), which has a glass transition temperature of $42^{\circ} \mathrm{C}$. The ingredients used are listed in Table 1.

The sodium dodecyl sulphate and 1-octanol were dissolved in $40 \mathrm{ml}$ of distilled water using a magnetic stirrer, and later $50 \mathrm{mg}$ of $\mathrm{CeGdZn}$-ferrite nanoparticles were added. The polymer phase was prepared by dissolving $2 \mathrm{gm}$ of ethyl cellulose into $10 \mathrm{ml}$ of methylene chloride. A crude emulsion was formed by adding the polymer phase to the medium aqueous phase. The crude emulsion was sonicated using an ultrasonicator five times in steps of 3 minutes. The resultant emulsion was then stirred inside a round bottom flask for 12 hours at $700 \mathrm{rpm}$. The solvent was then removed using a vacuum evaporation method. The polymer-encapsulated particles formed were washed with acetone and stored under the PBS buffer solution.

2.3. Magnetic Heating. The apparatus used in estimating the magnetic heating depicted similarity to the coil devised by Jordan's. The existence of a small difference in other setups prevented sample heating from the coil by running water through the coils. However, in this study, the foam and apparatus created a homogeneous alternating magnetic field within the coil, which in turn created E-fields in space perpendicular to the magnetic field at the center of the coil. $\mathrm{X}$-ray diffraction is used to determine the identity of crystalline solids based on their atomic Plexiglas that prevented heat from transferring to the sample from the coil. Insulation also served to maintain heat within the sample to prevent excessive air cooling of the sample. This apparatus created a homogeneous alternating magnetic field within the coil, which in turn created E-fields in space perpendicular to the magnetic field at the center of the coil. X-ray diffraction is used to determine the identity of crystalline solids based on their atomic structure. During X-ray diffraction analysis,
TABLE 1: Ingredients for ethyl cellulose-encapsulated CeGdZnFe using polymer emulsion.

\begin{tabular}{lc}
\hline Ingredients & Quantity \\
\hline Polymer phase: ethyl cellulose & $2 \mathrm{gm}$ \\
Solvent: methylene chloride & $10 \mathrm{ml}$ \\
Aqueous medium phase: water & $40 \mathrm{ml}$ \\
Emulsifying agent: sodium dodecyl & $0.2 \mathrm{gm}$ \\
sulphate & $0.5 \mathrm{ml}, 0.4 \mathrm{gm}$ \\
Inhibitor compound: 1-octanol & $50 \mathrm{mg}$ \\
Magnetic particles: CeGdZn-ferrite & $1: 40$ (approximately) \\
Magnetic particle: Polymer ratio &
\end{tabular}

X-ray beams are reflected off the parallel atomic layers within the nanoparticle material over a range of diffraction angles.

Magnetic susceptibility is a dimensionless proportionality constant that describes the degree of magnetization of a material in response to the external magnetic field, it is the ratio of magnetization $M$ to the field $H$. When subjected to an alternating current (AC) magnetic field, the magnetization of nanoparticles may not be able to follow the AC field due to its finite rate of magnetic relaxation, thus a phase delay between the AC field and the magnetization is introduced. This property introduces a complex magnetic susceptibility, which can be calculated by the Debye model. Magnetic susceptibility holds great significance in magnetic hyperthermia applications. Usually, in magnetic hyperthermia models, magnetic properties such as the saturation magnetization are considered to be constant with temperature. Magnetic saturation is the state reached when an increase in the applied external magnetic field $H$ cannot increase the magnetization of the material further, so the total magnetic flux density $B$ levels off although it continues to increase very slowly due to the vacuum permeability. Saturation is a characteristic of ferromagnetic and ferrimagnetic materials and their alloys. In magnetic hyperthermia experiments, an alternating magnetic field is applied to the nanoparticle sample and the variation of temperature is measured. The heating efficiency is represented by the specific absorption rate which is usually obtained from the initial slope of the measured data.

\subsection{TEM Magnetic CeGdZn-Ferrite Particles Characterization.} Transmission electron microscopy (TEM) is used to characterize the morphology of materials such as nanoparticles. The samples are prepared for TEM imaging by inserting a TEM grid (copper coated with formvar) into a dry or wet powder. The powder is usually dried overnight using tweezers to hold the grid. The sample grid is then lightly tapped to remove any excess particles, and the grid is placed in the TEM for imaging. This procedure can be used to characterize the coated magnetic particles. TEM samples were prepared by placing a drop of the suspensions on a copper grid with a carbon membrane film. Images were taken using JEOL-2010 TEM operating up to $200 \mathrm{kV}$. 
2.5. DC Superconducting Quantum Interference Device (SQUID) Analysis. The DC SQUID magnetometer was used to characterize the magnetic properties of superparamagnetic nanoparticle systems. Using this device, $\pm 5 \mathrm{~T}$ DC fields can be applied to samples from $5 \mathrm{~K}$ to $400 \mathrm{~K}$, with a sensitivity of $1 \times 10^{-7}$ EMU in the detected moment. A SQUID is the most sensitive device available for measuring magnetic fields. It does not detect the magnetic field directly from the sample although the SQUID in the MPMS is the source of the instrument's remarkable sensitivity. Instead, the sample moves through a system of superconducting detection coils, which are connected to the SQUID with superconducting wires allowing the current from the detection coils to inductively coupled to the SQUID sensor.

2.6. Curie Temperature. A major distinction between ferromagnets and paramagnets is that the ferromagnetic state is of the long-range order. This long-range order sets in at a phase transition, which occurs at the Curie temperature. The Curie temperature is close to the same point where a $(1 / \chi$ vs. T) plot, $\chi$ being the susceptibility, extrapolates to zero for a Curie-Weiss paramagnet that becomes ferromagnetic. The optimum heating range reaches $42-43^{\circ} \mathrm{C}$, if the material of the magnetic nanoparticles has Curie temperature. If they are further subjected to the magnetic field of any intensity, they will not be heated up as the nanoparticles become paramagnetic.

\section{Results and Discussion}

3.1. Powder XRD Pattern. The physical properties of assynthesized CeGdZn-ferrite nanoparticles were shown to depend on their size. Prepared nanoparticles demonstrated a certain polydispersity. It seemed interesting to compare the properties of small and large particles fractionated from the same polydisperse sample. The crystalline structure of the samples was identified from X-ray diffraction (XRD) patterns recorded in the $2 \theta$ range $10-90^{\circ}$ with a scan step of $0.05^{\circ}(2 \theta)$ for $5 \mathrm{~s}$ on a Philips X'pert Pro diffractometer, $\mathrm{Cu}$ $\mathrm{K} \alpha$ radiation. The crystallite size was determined from the different diffraction peaks $(h k l)$, using the Scherrer method. The XRD pattern for the CeGdZn-ferrite magnetic nanoparticles is shown in Figure 2.

3.2. TEM Characterization. Figure 3 shows the TEM micrograph of the dispersion of CeGdZn-ferrite nanoparticles and CeGdZn-ferrite ethyl cellulose encapsulated nanoparticles in ethanol deposited over a $\mathrm{Cu}$ grid. The sample consists of a dispersion of almost spherical particles with a narrow size distribution. The histogram of the diameter has been obtained from a statistical analysis of over 100 particles.

Table 2 lists the main structural features of the prepared CeGdZn-ferrite nanoparticles. The table includes the mean radii obtained by both XRD and TEM methods, concentration per flask, crystalline structure, and surface chemistry.
3.3. Magnetic Heating Tests and Results. The heating pattern for CeGdZn-ferrite magnetic nanoparticles is shown in Figure 4 . The results have shown the temperature rise as an effect of the ferromagnetic sample. The results need to be stressed in terms of temperature rise overheating because of the following two reasons:

(i) The likelihood of RF interference contributing to an apparent increase in temperature

(ii) The inability to completely characterize heat-producing mechanisms in this specific experimental setting

Various procedures need to be attempted to show the contributions of the first reason by using the proper control materials. Also, the second reason has been discussed in terms of current research to help justify claims of more pronounced temperature rises in ferromagnetic materials.

From the hysteresis loop, primary magnetic properties, such as retentivity and coercive force of a material, can be determined. Retentivity, the material's ability to retain a certain amount of residual magnetic field when the magnetizing force is removed after achieving saturation, depicts the value of $B$ axis on the hysteresis curve. Coercive force, the amount of reverse magnetic field which must be applied to a magnetic material to make the magnetic flux return to zero, depicts the value of $H$ axis on the hysteresis curve. Coercivity and retentivity of nanoparticles have been found to increase with a decrease in temperature of the samples. It has been observed that the coercivity and retentivity follow a simple model of thermal activation of particle's moment over the anisotropy barrier in the temperature range (5$300 \mathrm{~K}$ ), in accordance with Kneller's law for ferromagnetic materials.

The heating pattern for CeGdZnFe [Gd, $x=0.2$ conc., $20 \mathrm{mg} / \mathrm{ml}$ ] using an alcohol thermometer at $963 \mathrm{kHz}$ is depicted in Figure 5.

3.4. Curie Temperature Testing. It is a well-known fact that it is difficult to determine the Curie temperature (Tc) of a ferromagnetic body accurately [38]. These difficulties are incurred because the curve of the spontaneous magnetization versus temperature ( $\sigma$ vs. Tc) does not intersect the temperature axis steeply, but instead bends over to form an asymptotic tail. The temperature defined by extrapolation of the central part of the $\sigma(\mathrm{T})$ curve is called the ferromagnetic Curie temperature, Tc. There is a simple, inexpensive, accurate, and fast susceptibility method to measure Tc, which has been in use for a long time. In this method, a ferromagnetic sample serves as a core of a linear variable differential transformer (LVDT). Changes in the LVDT output due to changes in sample susceptibility may be detected electronically. Electronic circuitry accurately detects when both the ferromagnetic moment of a sample and the sample susceptibility approach becomes zero with increasing temperature. This temperature may be defined as the Curie temperature. The setup for Curie temperature testing for various nanoparticles includes forward power $=40 \mathrm{~W}$ that is similar to load power since the equipment is at resonance. It 


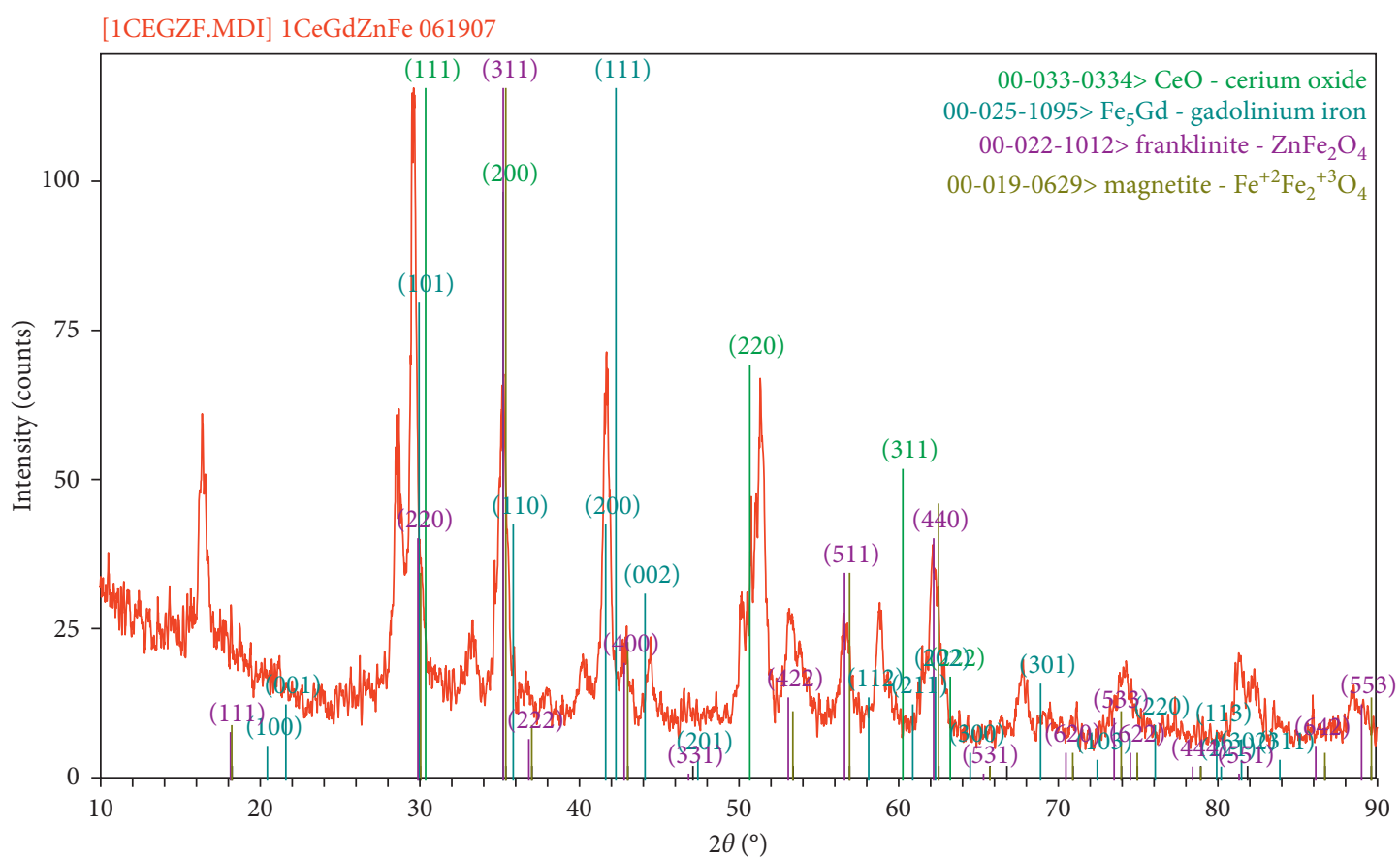

(a)

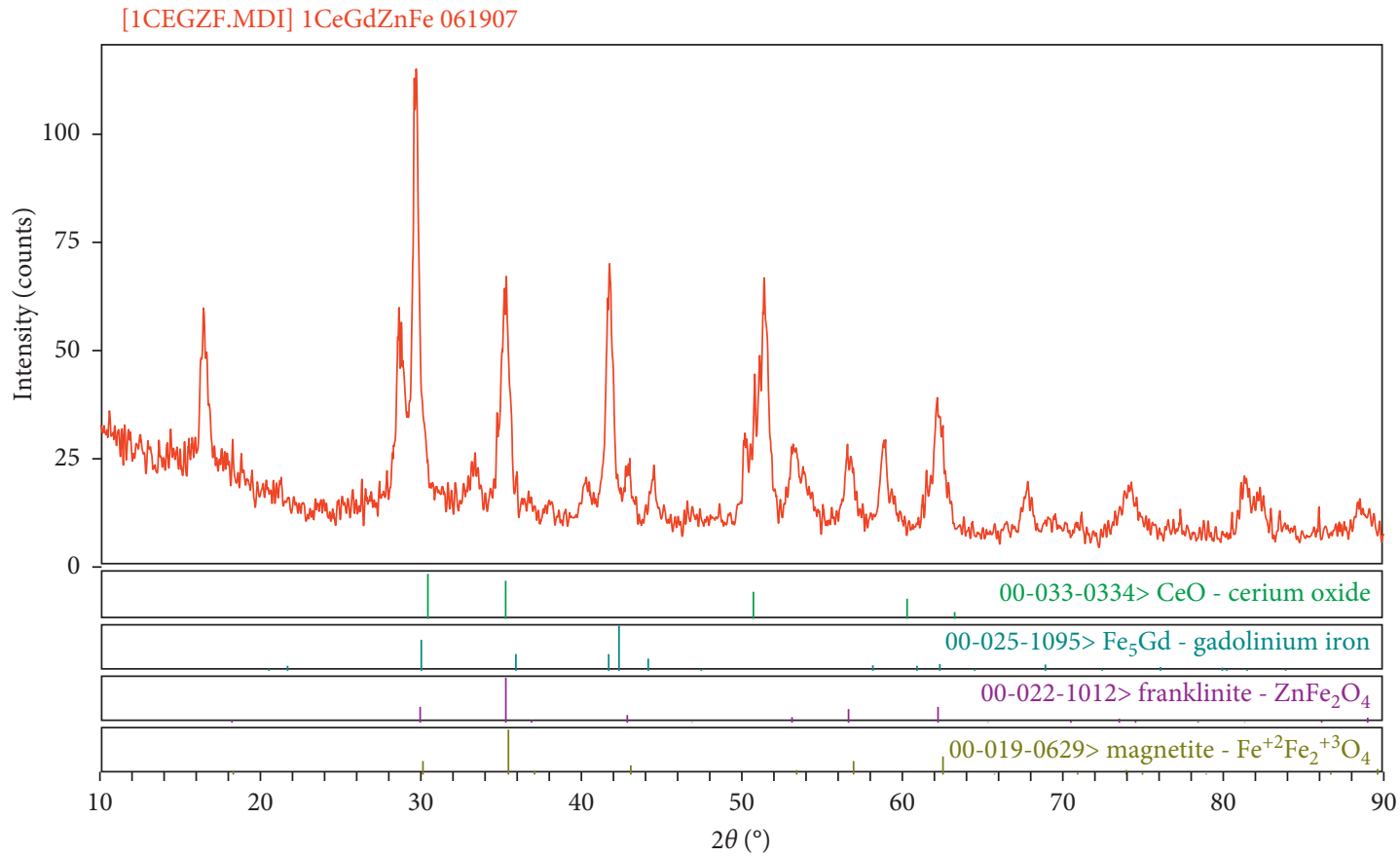

(b)

Figure 2: Powder X-ray diffraction pattern of and reference pattern for CeGdZn-ferrite magnetic nanoparticles. The pattern is representative for all the samples of the series.

also required a function generator and power amplifier tested at $V=30 \mathrm{~V}$ peak-to-peak and a frequency of $961 \mathrm{kHz}$ at coil resonance. The Curie temperature results with $x$ of 0 , $0.5,1$, and 1.5 were evaluated, which showed that Tc varies with the Gd concentration changes that ferrite NPs Tc can be tuned. The Curie temperature was tuned for Mn ferrite with $\mathrm{Zn}$ and $\mathrm{Zn}$ ferrite with $\mathrm{Gd}$ ranging from $42^{\circ} \mathrm{C}$ to $43^{\circ} \mathrm{C}$.
Apostolov et al. [39] have shown that this tuning is suitable to be applied for hyperthermia. A one-minute break between each run was observed to help prevent dominant coil heating rather than nanoparticle heating. Also, no cool down via a water-filled tube or glove was adhered to reach higher temperatures. It tends to utilize the remnant heat produced by nanoparticles as well as the coil. 


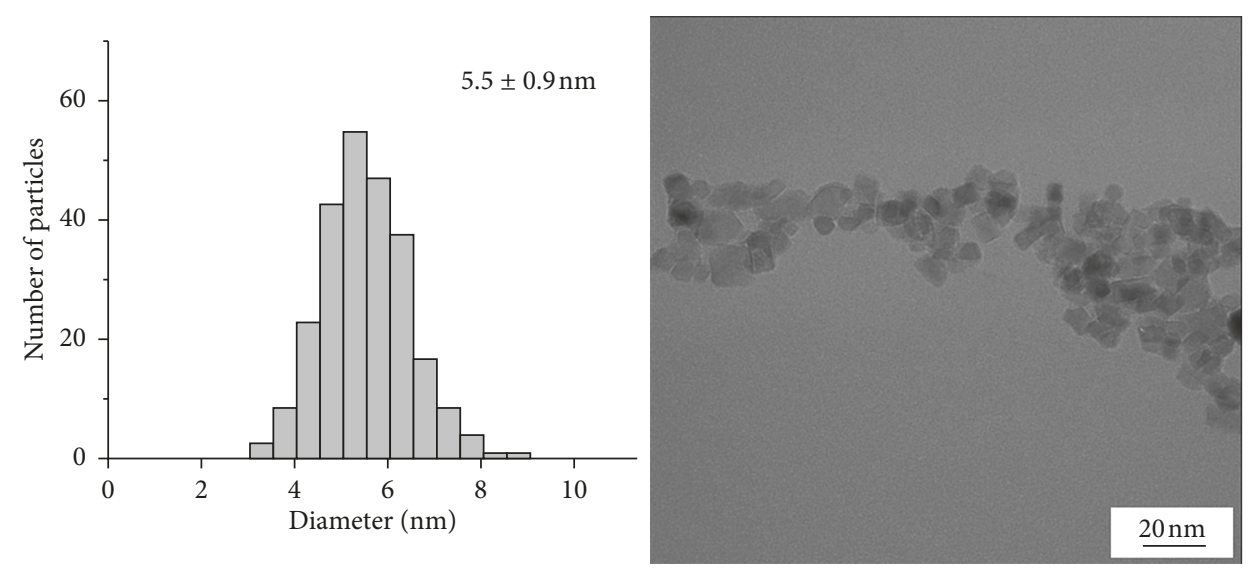

FIGURE 3: Ethyl cellulose encapsulated CeGdZn-ferrite by polymer emulsion.

Table 2: Characteristics of the CeGdZn-ferrite inorganic nanoparticles used in experimentation.

\begin{tabular}{|c|c|c|c|c|c|}
\hline Sample & Mean radius DXRD $(\mathrm{nm})$ & Mean radius DTEM $(\mathrm{nm})$ & Concentration per flask $(\mathrm{g} / \mathrm{mL})$ & Crystalline structure & Surface chemistry \\
\hline 1 & $15 \pm 0.4$ & $11 \pm 0.4$ & $3.303 \times 10^{-2}$ & Crystalline & Hydrophilic \\
\hline 2 & $13 \pm 0.5$ & $9 \pm 0.5$ & $2.180 \times 10^{-3}$ & Crystalline & Hydrophilic \\
\hline 3 & $10 \pm 0.2$ & $6 \pm 0.7$ & $1.130 \times 10^{-4}$ & Crystalline & Hydrophilic \\
\hline 4 & $8 \pm 0.5$ & $5 \pm 0.2$ & $3.201 \times 10^{-2}$ & Crystalline & Hydrophilic \\
\hline
\end{tabular}

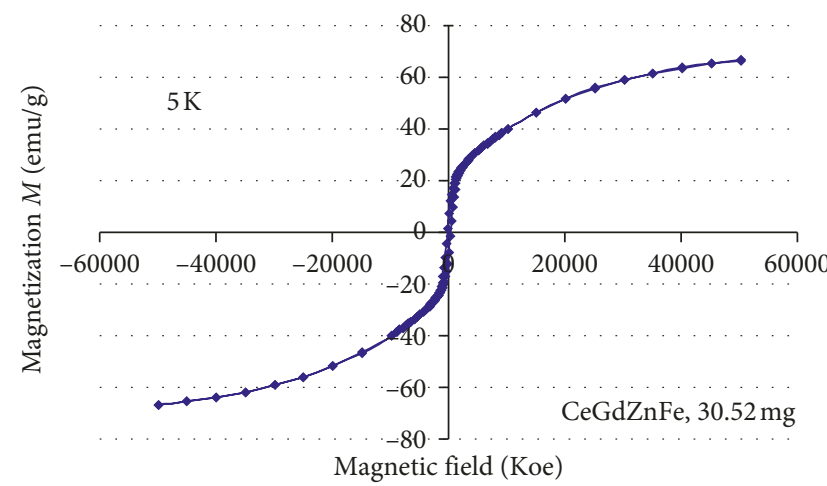

(a)

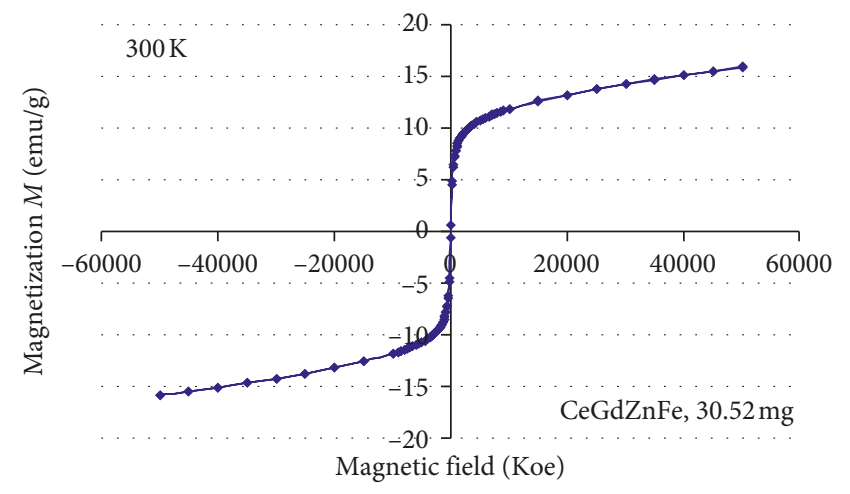

(b)

FIgURE 4: CeGdZn-ferrite nanoparticles magnetization curve taken by SQUID at $5 \mathrm{~K}$ (a) and at $300 \mathrm{~K}$ (b).

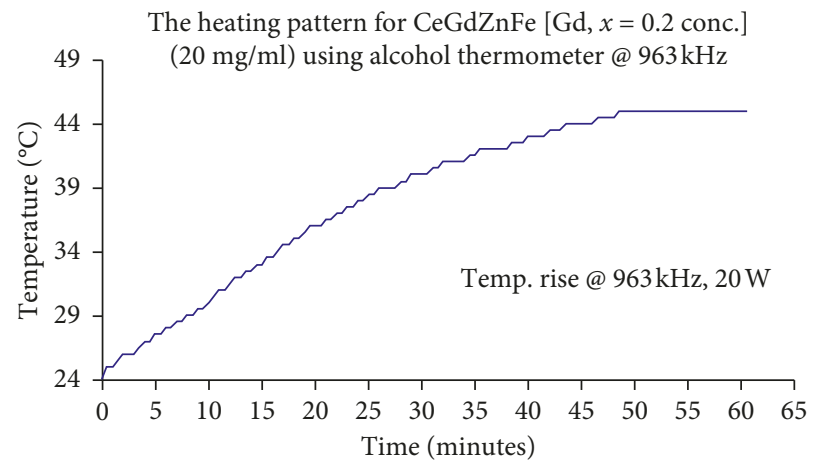

FIGURE 5: The heating pattern of CeGdZn-ferrite [Gd, $x=0.2$ conc.] $(20 \mathrm{mg} / \mathrm{ml})$ at $963 \mathrm{kHz}$. 


\section{Conclusion}

The study showed the difference in the decayed ferromagnetism based on the difference of the magnetic material concerning the magnetic disordering process in the vicinity of Tc. Diminishing returns on temperature rise in CeGdZnferrite nanoparticles should occur near Tc. This causes a decrease in temperature for CeGdZn-ferrite nanoparticles as the starting temperature of tests increases towards Tc due to the ferromagnetic gradual loss of magnetization, given that losses and heat production are considered a result of hysteresis. Understanding ferromagnetic behavior above the body temperature will help to find if particles can heat to hyperthermia temperatures and self-limit near the Curie temperature. Cerium oxide nanoparticles with integrated gadolinium, exhibit combined therapeutic and diagnostic capabilities. This type of nanomaterial is highly promising for applications in the fields of biomedical imaging and magnetic hyperthermia.

\section{Data Availability}

The data used to support the findings of this study are available from the corresponding author upon request.

\section{Conflicts of Interest}

The author declares that there are no conflicts of interest regarding the publication of this paper.

\section{Acknowledgments}

The author would like to acknowledge the financial support for this work from the Deanship of Scientific Research (DSR), University of Tabuk, Saudi Arabia, under grant no. S-0163-1436.

\section{References}

[1] H. J. Kwon, K. Shin, M. Soh et al., "Large-scale synthesis and medical applications of uniform-sized metal oxide nanoparticles," Advanced Materials, vol. 30, no. 42, 2018.

[2] I. Obaidat, B. Issa, and Y. Haik, "Magnetic properties of magnetic nanoparticles for efficient hyperthermia," Nanomaterials, vol. 5, no. 1, pp. 63-89, 2015.

[3] H. Hejase, S. S. Hayek, S. Qadri, and Y. Haik, "MnZnFe nanoparticles for self-controlled magnetic hyperthermia," Journal of Magnetism and Magnetic Materials, vol. 324, no. 22, pp. 3620-3628, 2012.

[4] E. C. Abenojar, S. Wickramasinghe, J. Bas-Concepcion, and A. C. S. Samia, "Structural effects on the magnetic hyperthermia properties of iron oxide nanoparticles," Progress in Natural Science: Materials International, vol. 26, no. 5, pp. 440-448, 2016.

[5] S. V. Jadhav, B. M. Kim, H. Y. Lee et al., "Induction heating and in vitro cytotoxicity studies of $\mathrm{MnZnFe}_{2} \mathrm{O}_{4}$ nanoparticles for self-controlled magnetic particle hyperthermia," Journal of Alloys and Compounds, vol. 745, pp. 282-291, 2018.

[6] S. M. North and T. A. Banks, Small Animal Oncology E-Book: An Introduction, Elsevier Health Sciences, London, UK, 2009.

[7] P.-E. Le Renard, O. Jordan, A. Faes et al., "The in vivo performance of magnetic particle-loaded injectable, in situ gelling, carriers for the delivery of local hyperthermia," Biomaterials, vol. 31, no. 4, pp. 691-705, 2010.

[8] R. K. Gilchrist, R. Medal, W. D. Shorey, R. C. Hanselman, J. C. Parrott, and C. B. Taylor, "Selective inductive heating of lymph nodes," Annals of Surgery, vol. 146, no. 4, pp. 596-606, 1957.

[9] V. Bessalova, N. Perov, and V. Rodionova, "New approaches in the design of magnetic tweezers-current magnetic tweezers," Journal of Magnetism and Magnetic Materials, vol. 415, pp. 66-71, 2016.

[10] P.-E. L. Renard, F. Buchegger, A. Petri-Fink et al., "Local moderate magnetically induced hyperthermia using an implant formed in situ in a mouse tumor model," International Journal of Hyperthermia, vol. 25, no. 3, pp. 229-239, 2009.

[11] Q. A. Pankhurst, J. Connolly, S. K. Jones, and J. Dobson, "Applications of magnetic nanoparticles in biomedicine," Journal of Physics D: Applied Physics, vol. 36, no. 13, pp. R167-R181, 2003.

[12] A. A. Kuznetsov, O. A. Shlyakhtin, N. A. Brusentsov, and O. A. Kuznetsov, "Smart mediators for self-controlled inductive heating," European cells \& materials, vol. 3, no. 2, pp. 75-77, 2002.

[13] A. Jordan, R. Scholz, P. Wust, H. Fähling, and R. Felix, "Magnetic fluid hyperthermia (MFH): cancer treatment with AC magnetic field induced excitation of biocompatible superparamagnetic nanoparticles," Journal of Magnetism and Magnetic Materials, vol. 201, no. 1-3, pp. 413-419, 1999.

[14] A. J. Giustini, A. A. Petryk, S. M. Cassim, J. A. Tate, I. Baker, and P. J. Hoopes, "Magnetic nanoparticle hyperthermia in cancer treatment," Nano Life, vol. 1, no. 2, pp. 17-32, 2010.

[15] S. H. A. Al Lehyani, R. A. Hassan, A. A. Alharbi, T. Alomayri, and H. Alamri, "Magnetic hyperthermia using cobalt ferrite nanoparticles: the influence of particle size," International Journal of Advancements in Technology, vol. 8, no. 4, 2017.

[16] M. Lahonian, "Diffusion of magnetic nanoparticles within a biological tissue during magnetic fluid hyperthermia," in Hyperthermia, InTechOpen, London, UK, 2013.

[17] A. Kmita, D. Lachowicz, J. Żukrowski et al., "One-step synthesis of long term stable superparamagnetic colloid of zinc ferrite nanorods in water," Materials, vol. 12, no. 7, p. 1048, 2019.

[18] S. Ullrich, S. P. Scheeler, C. Pacholski, J. P. Spatz, and S. Kudera, "Colloidal nanoparticles: formation of large $2 \mathrm{D}$ arrays of shape-controlled colloidal nanoparticles at variable interparticle distances (part. Part. Syst. Charact. 1/2013)," Particle \& Particle Systems Characterization, vol. 30, no. 1, p. 2, 2012.

[19] A. Singh, A. Singh, S. Singh, P. Tandon, B. C. Yadav, and R. R. Yadav, "Synthesis, characterization and performance of zinc ferrite nanorods for room temperature sensing applications," Journal of Alloys and Compounds, vol. 618, pp. 475-483, 2015.

[20] T. Ahmad, H. Bae, Y. Iqbal et al., "Chitosan-coated nickelferrite nanoparticles as contrast agents in magnetic resonance imaging," Journal of Magnetism and Magnetic Materials, vol. 381, pp. 151-157, 2015.

[21] S. Manjura Hoque, C. Srivastava, N. Venkatesha, P. S. Anil Kumar, and K. Chattopadhyay, "Superparamagnetic behaviour and $\mathrm{T} 1, \mathrm{~T} 2$ relaxivity of $\mathrm{ZnFe}_{2} \mathrm{O}_{4}$ nanoparticles for magnetic resonance imaging," Philosophical Magazine, vol. 93, no. 14, pp. 1771-1783, 2013.

[22] S. Xavier, M. K. Jiji, S. Thankachan, and E. M. Mohammed, "Effect of sintering temperature on the structural and 
electrical properties of cobalt ferrite nanoparticles," in Proceedings of the AIP Conference, USA, September 2014.

[23] Z. Zhou, X. Zhu, D. Wu et al., "Anisotropic shaped iron oxide nanostructures: controlled synthesis and proton relaxation shortening effects," Chemistry of Materials, vol. 27, no. 9, pp. 3505-3515, 2015.

[24] S. M. Hoque, M. S. Hossain, S. Choudhury, S. Akhter, and F. Hyder, "Synthesis and characterization of $\mathrm{ZnFe}_{2} \mathrm{O}_{4}$ nanoparticles and its biomedical applications," Materials Letters, vol. 162, pp. 60-63, 2016.

[25] T. Guo, M. Lin, J. Huang et al., "The recent advances of magnetic nanoparticles in medicine," Journal of Nanomaterials, vol. 2018, Article ID 7805147, 8 pages, 2018.

[26] S. Laurent, S. Dutz, U. O. Häfeli, and M. Mahmoudi, "Magnetic fluid hyperthermia: focus on superparamagnetic iron oxide nanoparticles," Advances in Colloid and Interface Science, vol. 166, no. 1-2, pp. 8-23, 2011.

[27] M. B. Lilly, I. A. Brezovich, and W. J. Atkinson, "Hyperthermia induction with thermally self-regulated ferromagnetic implants," Radiology, vol. 154, no. 1, pp. 243-244, 1985.

[28] M. Kallumadil, M. Tada, T. Nakagawa, M. Abe, P. Southern, and Q. A. Pankhurst, "Suitability of commercial colloids for magnetic hyperthermia," Journal of Magnetism and Magnetic Materials, vol. 321, no. 10, pp. 1509-1513, 2009.

[29] C. F. Kernizan, K. J. Klabunde, C. M. Sorensen, and G. C. Hadjipanayis, "Magnetic properties of nanometer-scale iron particles generated by iron atom clustering in cold pentane," Chemistry of Materials, vol. 2, no. 1, pp. 70-74, 1990.

[30] J. P. Chen, K. M. Lee, C. M. Sorensen, K. J. Klabunde, and G. C. Hadjipanayis, "Magnetic properties of microemulsion synthesized cobalt fine particles," Journal of Applied Physics, vol. 75, no. 10, pp. 5876-5878, 1994.

[31] D. Vollath, D. V. Szabó, R. D. Taylor, and J. O. Willis, "Synthesis and magnetic properties of nanostructured maghemite," Journal of Materials Research, vol. 12, no. 8, pp. 2175-2182, 1997.

[32] H. I. Schlesinger, H. C. Brown, H. R. Hoekstra, and L. R. Rapp, "Reactions of diborane with alkali metal hydrides and their addition compounds. New syntheses of borohydrides. Sodium and potassium borohydrides1," Journal of the American Chemical Society, vol. 75, no. 1, pp. 199-204, 1953.

[33] L. Yiping, G. C. Hadjipanayis, C. M. Sorensen, and K. J. Klabunde, "Magnetic and structural properties of ultrafine Co-B particles," Journal of Magnetism and Magnetic Materials, vol. 79, no. 3, pp. 321-326, 1989.

[34] S. Wells, S. W. Charles, S. Mørup et al., "A study of Fe-B and Fe-Co-B alloy particles produced by reduction with borohydride," Journal of Physics: Condensed Matter, vol. 1, no. 43, pp. 8199-8208, 1989.

[35] K. S. Suslick, T. Hyeon, and M. Fang, "Nanostructured materials generated by high-intensity ultrasound: sonochemical synthesis and catalytic studies," Chemistry of $\mathrm{Ma}$ terials, vol. 8, no. 8, pp. 2172-2179, 1996.

[36] Q. Wang, H. Xia, and C. Zhang, "Preparation of polymer/ inorganic nanoparticles composites through ultrasonic irradiation," Journal of Applied Polymer Science, vol. 80, no. 9, pp. 1478-1488, 2001

[37] M. E. McHenry, S. A. Majetich, J. O. Artman, M. DeGraef, and S. W. Staley, "Superparamagnetism in carbon-coated co particles produced by the kratschmer carbon arc process," Physical Review B, vol. 49, no. 16, pp. 11358-11363, 1994.

[38] Q. Lin, J. Lin, Y. He, R. Wang, and J. Dong, "The structural and magnetic properties of gadolinium doped $\mathrm{CoFe}_{2} \mathrm{O}_{4}$ nanoferrites," Journal of Nanomaterials, vol. 2015, Article ID 294239, 6 pages, 2015.

[39] A. T. Apostolov, I. N. Apostolova, and J. M. Wesselinowa, "MO $\mathrm{Fe}_{2} \mathrm{O}_{3}$ nanoparticles for self- controlled magnetic hyperthermia," Journal of Applied Physics, vol. 109, no. 8, Article ID 083939, 2011. 


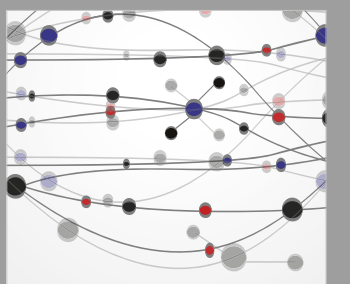

The Scientific World Journal
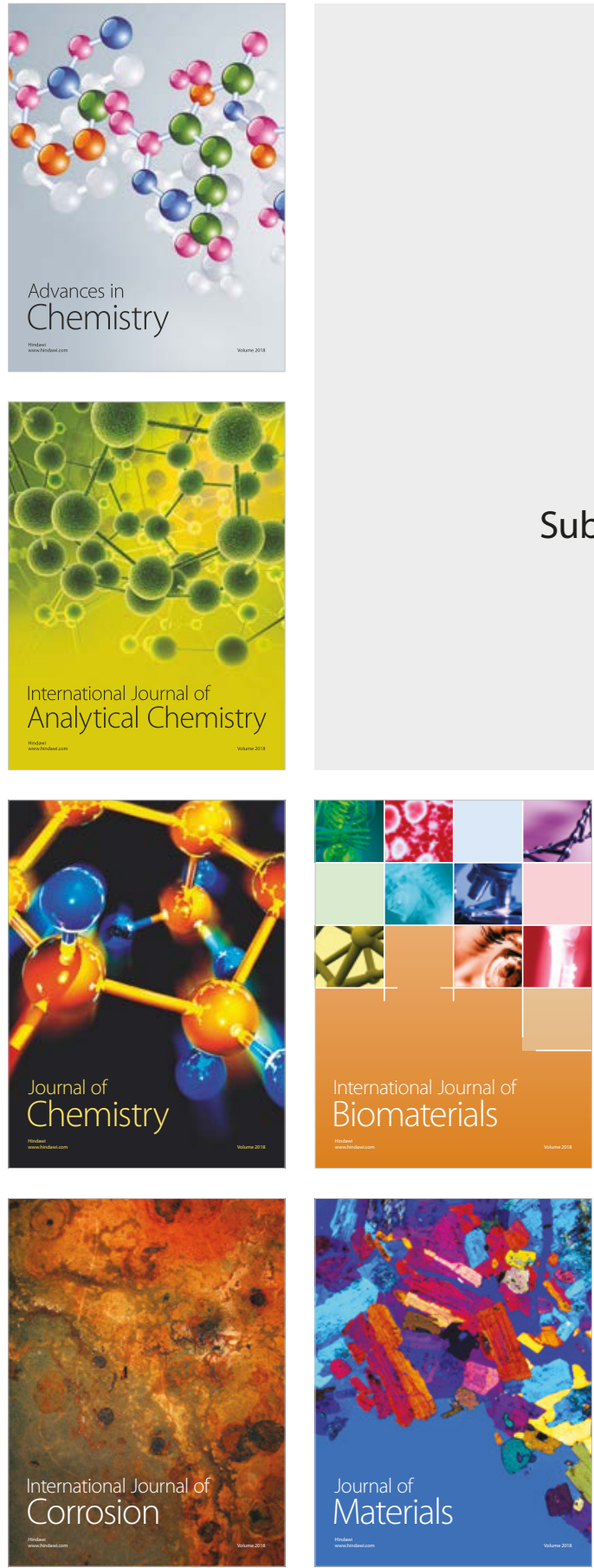

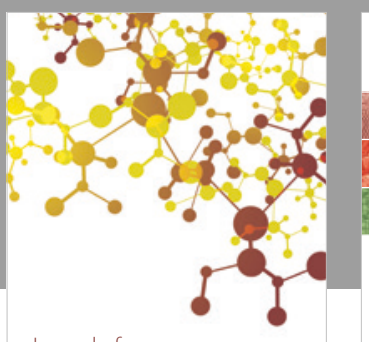

Journal of

Applied Chemistry
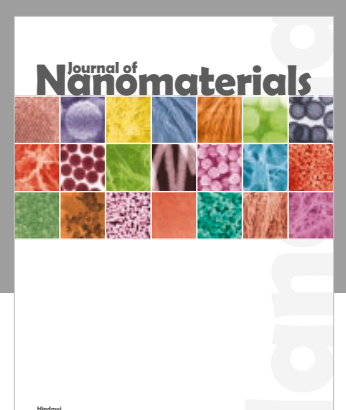

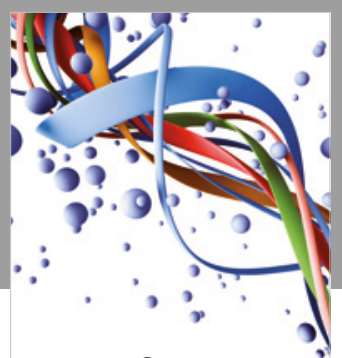

Scientifica

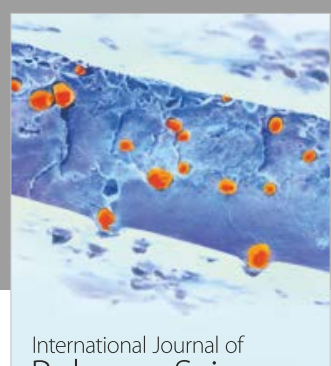

Polymer Science

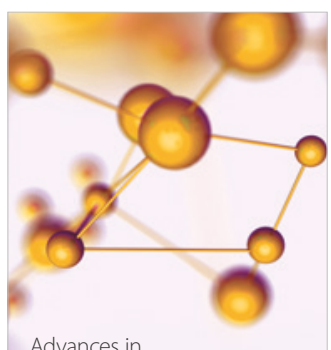

Physical Chemistry
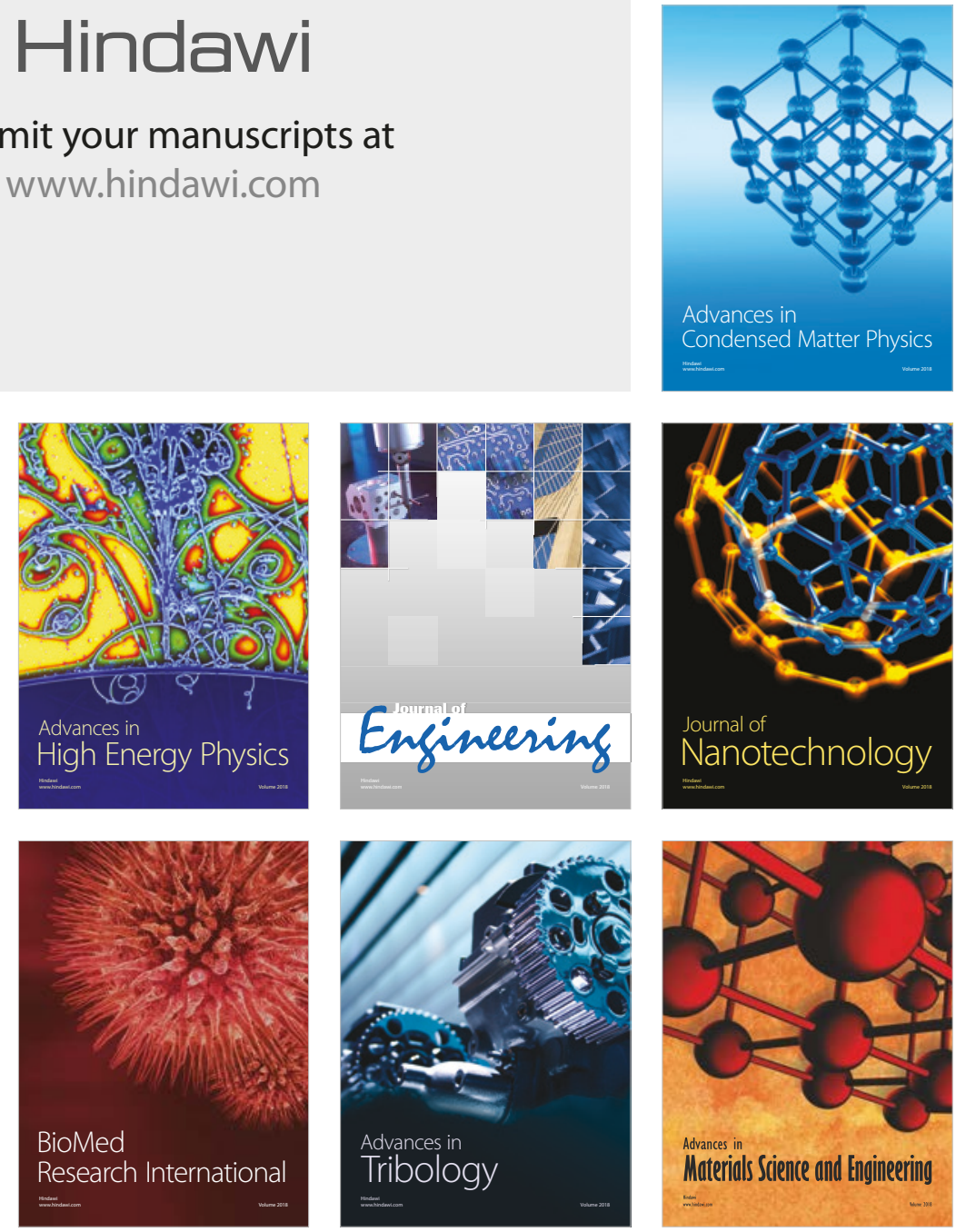